\title{
Propriedades mecânicas dos compósitos híbridos Vidro/Sisal
}

\section{Mechanical properties of Glass/Sisal hybrid composites}

\author{
Camila da Costa Barros ${ }^{1}$ \\ Tatiane Suyene da Paz Lacerda ${ }^{1}$ \\ Vitor Santos Lisboa ${ }^{1}$ \\ Shane Aparecida Goulart ${ }^{2}$ \\ Daniella Regina Mulinari ${ }^{2}$
}

\section{Artigo \\ Original \\ Original \\ Paper}

Recebido em 04/2011

Aprovado em $03 / 2012$

Neste trabalho foi avaliado o comportamento mecânico dos compósitos de poliéster reforçados com manta de vidro e tecido de sisal visando às necessidades da indústria automotiva na produção de componentes com menor custo e massa específica. Os compósitos foram moldados por compressão à temperatura ambiente e posteriormente curados à $60 \mathrm{oC}$ por 48 horas. Os resultados indicaram que houve um aumento nas propriedades mecânicas quando comparados aos compósitos processados por outros métodos com a mesma resina e reforço.

\section{Abstract}

In the work was evaluated the mechanical behaviour of the composites of polyester reinforced with glass mants and sisal fabric attend to necessities of automobile industry in the production of parts with smaller costs and specific mass. The composites were compression-molded at $25 \mathrm{oC}$ after cured to $60 \mathrm{oC}$ for 48 hours. Results showed an increase in the mechanical properties when compared to the composites processed for other methods with similar resin and reinforcement.
Key words:

Hybrid composites

Glass fibers

Sisal fibers 


\section{Introdução}

Atualmente, a necessidade de desenvolver e comercializar materiais compósitos reforçados com fibras naturais tem crescido, devido a questões relacionadas ao desenvolvimento sustentável (Silva, C. D. et al., 2004; Vieira, C. A. B., 2008). Outra razão é a vasta possibilidade de modelagem e a obtenção de peças de design com respaldo ambiental (Alves, $\mathrm{C}$, et al., 2010; Bakare, I. O., et al., 2010).

Buscando-se uma melhoria na tecnologia de novos materiais para substituir adequadamente as fibras sintéticas por uma matéria-prima proveniente de fonte renovável, vários pesquisadores têm desenvolvido compósitos poliméricos reforçados com fibras naturais (Mulinari, D. R., et al., 2009; Wambua, P., et al., 2003).

Embora as fibras naturais apresentem resistência mecânica inferior às fibras sintéticas, as mesmas exibem propriedades mecânicas específicas que as permitem serem utilizadas como reforço em matrizes poliméricas (Ibrahim, M. M., et al., 2010).

Compósitos poliméricos reforçados com fibras naturais possuem potencial para aplicação em componentes sujeitos a carregamentos leves ou moderados. As principais aplicações estão na construção civil, indústria de móveis, embalagens e no ramo automotivo (Mulinari, D. R., 2009). O baixo comportamento mecânico e alta absorção de umidade dessas fibras são os grandes empecilhos para novas aplicações (Chow, $\mathrm{C}$. P. L., et al., 2007; Stark, N., 2001). Uma alternativa para minimizar este problema é o processo de hibridização, associando fibras naturais e sintéticas (Da Silva, R. V., et al., 2008).

A fibra de vidro é uma boa indicação para a hibridização, pois evidencia uma relação favorável entre custo e comportamento mecânico. Além disso, pode atuar como barreira química prevenindo o contato das fibras naturais, naturalmente hidrofílicas, com a água ( $\mathrm{Da}$ Silva, R. V., et al., 2008).

No entanto, esses compósitos podem manifestar vários tipos de falhas quando submetidos a carregamento cíclicos, incluindo delaminação, falha da matriz e fratura na fibra. Durante o carregamento cíclico, o comportamento de fratura de compósitos pode ser influenciado por parâmetros como a interface fibra/polímero, método de processamento do compósito e arquitetura das fibras, contudo, é importante considerar o forte efeito das fibras na redistribuição de tensão no material, sendo a deformação da matriz um parâmetro também fundamental para a fadiga do material (Ray, D., et al., 2002; Yuanjian, T., et al., 2007).

Nesse contexto, novos materiais têm surgido como alternativas para melhorar o desempenho estrutural dos compósitos poliméricos reforçados com fibras naturais (Gerrard, J., et al., 2007; Sreekumar, P.A., et al., 2007).

Dessa forma, a proposta deste trabalho foi desenvolver e avaliar o comportamento mecânico do compósito híbrido de poliéster reforçado com mantas de vidro e tecido de sisal.

\section{Materiais e Métodos}

\subsection{Materiais}

A resina poliéster utilizada neste trabalho foi a ortoftálica insaturada comercial 430-C NOVAPOL, de Serra-ES. Trata-se de uma resina rígida, de alta viscosidade e baixa reatividade, pré acelerada com densidade entre 1,10 e 1,15 g.cm-3 $\left(25^{\circ} \mathrm{C}\right)$. A razão estequiométrica de resina/iniciador utilizada para a reação de cura foi de 100 partes em volume de resina poliéster para 20 partes em volume de peróxido de metil-etil-cetona (PMEK). O reforço sugerido para o projeto foi a de fibra de sisal em forma de tecido e a fibra de vidro (manta de fibras curtas com $450 \mathrm{~g} / \mathrm{m}^{2}$ ).

\subsection{Obtenção dos Laminados}

O processamento dos laminados foi feito utilizando moldagem por compressão sob 1,5 toneladas por 1 minuto, conforme Figura 1.

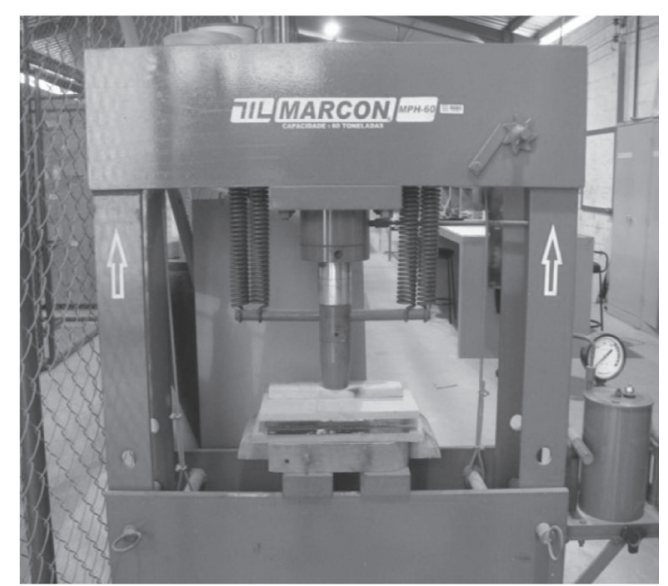

Figura 1. Processo utilizado para obtenção do laminado. 
A espessura do laminado foi a equivalente a uma camada de tecido. A configuração do laminado híbrido é mostrada na Figura 2.

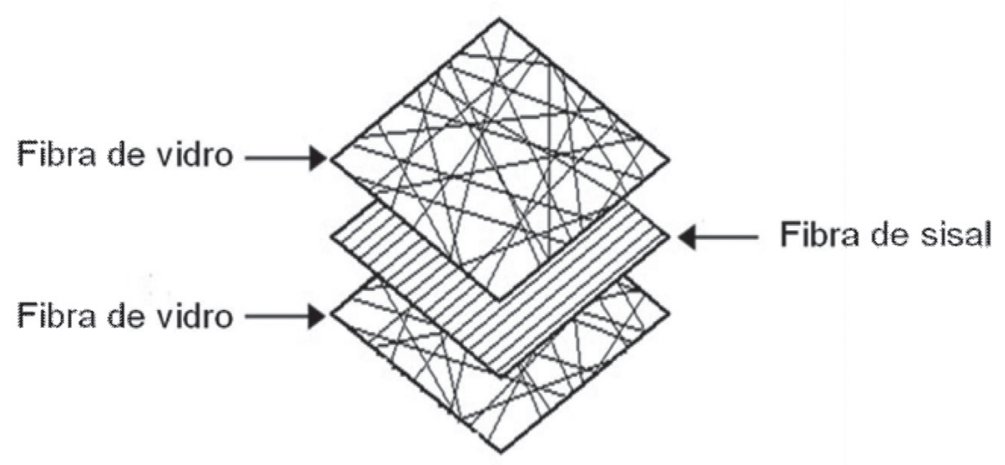

Figura 2. Configuração do laminado híbrido vidro/sisal.

Todo o processo foi realizado à temperatura ambiente e a desmoldagem dos laminados com resina ocorreu um dia após a moldagem, respectivamente. Como desmoldante para os laminados foram utilizadas folhas plásticas. Em seguida, os laminados foram curados em estufa a $60{ }^{\circ} \mathrm{C}$ por 48 horas. A espessura do laminado foi a equivalente a uma camada de tecido. O mesmo procedimento foi repetido para o laminado feito somente de mantas de vidro. A densidade dos laminados foi determinada segundo a norma ASTM D792-91e a fração volumétrica dos compósitos híbridos foi $36 \%$, tipicamente $13 \%$ de fibra de sisal e $23 \%$ de fibra de vidro.

\subsection{Propriedades Mecânicas}

As propriedades mecânicas foram avaliadas por meio de ensaios mecânicos de tração, flexão e impacto.

Os ensaios de tração e flexão foram realizados no Laboratório de Ensaios Mecânicos da Escola de Engenharia de Lorena - USP, em um equipamento da marca EMIC modelo DL10000. Para cada tipo de ensaio, foram ensaiados cinco corpos de prova (cdp's). Para os ensaios de tração os cdp's apresentaram dimensões de acordo com a norma ASTM D 3039. As propriedades mecânicas de resistência à tração, alongamento e módulo de elasticidade em tração foram avaliadas. Ensaios de flexão em três pontos foram realizados de acordo com a norma ASTM D790.

Os ensaios de impacto foram realizados no Laboratório de Ensaios Mecânicos do Centro Universitário de Volta Redonda UniFOA, utilizando o equipamento da marca PANTEC. Foram analisados cinco corpos de prova, com dimensões de acordo com a norma ASTM D 6110. Foram avaliadas a energia absorvida ao impacto e a resistência.

\section{Resultados E Discussão}

\subsection{Propriedades Mecânicas}

Os laminados híbridos apresentaram um comportamento linear até a fratura final; comum à maioria dos compósitos com matrizes termorrígidas e fibras sintéticas. Comportamento semelhante foi observado por Da Silva e colaboradores ao avaliar a resistência à flexão do laminado híbrido vidro/ curauá (Da Silva, R. V., et al., 2008). Amico e colaboradores também observaram o mesmo comportamento ao avaliar a resistência à flexão e a tração dos laminados híbridos vidro/ sisal (Amico, S. C., et al., 2010).

A Tabela 1 apresenta os valores do limite de resistência à flexão e à tração e seus respectivos alongamento e módulo, bem como a resistência ao impacto dos laminados híbridos. 
Tabela 1. Propriedades mecânicas dos laminados.

\begin{tabular}{|c|c|}
\hline Propriedades & Laminado Hibrido vidro/sisal \\
\hline Limite de resistência à flexão $(\mathrm{MPa})$ & $150,2 \pm 18,05$ \\
\hline Módulo de Elasticidade em flexão $(\mathrm{MPa})$ & $7700 \pm 951$ \\
\hline Alongamento no limite de resistência à flexão $(\%)$ & $8,5 \pm 0,5$ \\
\hline Limite de resistência à tração $(\mathrm{MPa})$ & $64 \pm 9,8$ \\
\hline Módulo de Elasticidade em tração $(\mathrm{MPa})$ & $4500 \pm 294,3$ \\
\hline Resistência ao Impacto $\left(\mathrm{kJ} / \mathrm{m}^{2}\right)$ & $113,8 \pm 42,8$ \\
\hline
\end{tabular}

Analisando-se os resultados da Tabela 1 com os dados encontrados na literatura, observaram-se resultados satisfatórios (Amico, S. C., et al., 2010; Cicala, G., et al., 2009).

Amico e colaboradores (2010) estudaram as propriedades mecânicas dos laminados híbridos vidro/sisal (v/s/v/s/v/s/v) com a mesma resina e obtiveram resultados inferiores aos obtidos neste trabalho. A resistência á flexão foi de $132 \mathrm{MPa}$ e a resistência ao impacto, $79 \mathrm{~kJ} / \mathrm{m}^{2}$. Comparando-se estes dados com os obtidos no neste trabalho, notou-se que o processamento escolhido e o número de camadas influenciaram no comportamento mecânico dos laminados. Este fato corrobora com os dados encontrados por Schmidt e colaboradores, os quais avaliaram as propriedades mecânicas dos laminados híbridos vidro/sisal (v/v/s/v/v) processados via moldagem por transferência de resina (RTM) (Schmidt, T. M., et al., 2009). A Tabela 2 evidencia as propriedades mecânicas obtidas por Schmidt e colaboradores.

Tabela 2. Propriedades mecânicas dos laminados híbridos (v/v/s/v/v) (Schmidt, T. M., et al., 2009).

\begin{tabular}{|c|c|}
\hline Propriedades & Laminado Hibrido vidro/sisal \\
\hline Limite de resistência à flexão $(\mathrm{MPa})$ & $181,1 \pm 17,7$ \\
\hline Módulo de Elasticidade em flexão $(\mathrm{MPa})$ & $8600 \pm 719$ \\
\hline Alongamento no limite de resistência à flexão $(\%)$ & $2,5 \pm 0,2$ \\
\hline Limite de resistência à tração $(\mathrm{MPa})$ & $62,6 \pm 3,6$ \\
\hline Módulo de Elasticidade em tração $(\mathrm{MPa})$ & $1330 \pm 199$ \\
\hline Resistência ao Impacto $\left(\mathrm{kJ} / \mathrm{m}^{2}\right)$ & $68,6 \pm 10,7$ \\
\hline
\end{tabular}

Analisando-se as Tabelas 1 e 2, observa-se uma redução na resistência à flexão e no módulo de elasticidade, de $20,5 \%$ e $11,7 \%$ respectivamente. Por outro lado, nota-se um aumento significativo na resistência ao impacto, e no módulo de elasticidade em tração, de $65,9 \%$ e $238,4 \%$ respectivamente. Este aumento ocorreu devido ao número de camadas e disposição das mesmas, bem como o processamento.

Dessa forma, pode-se afirmar que as condições de processamento para a obtenção destes compósitos foi satisfatória.

\section{Conclusões}

Os resultados obtidos nos ensaios mecânicos dos compósitos estudados demonstraram que os materiais possuem um comportamento linear até a fratura final, possibilitando a utilização da Lei de Hooke em qualquer formulação teórica. Os compósitos híbridos apresentaram propriedades mecânicas superiores aos encontrados na literatura com a mesma resina e fibras, demonstrando que as condições de processamento para a obtenção destes compósitos foi satisfatória. 


\section{Referências Bibliográficas}

1. VIEIRA, C. A. B. Avaliação de métodos de fabricação de mantas híbridas de fibras curtas de vidro e sisal em compósitos poliméricos. Dissertação (Mestrado em Ciência e Engenharia de Materiais) Universidade de Caxias do Sul, Caxias do Sul, 2008.

2. SANTOS, A. M. Estudo de compósitos híbridos polipropileno/ fibras de vidro e coco para aplicações em engenharia. Dissertação (Mestre em Engenharia Mecânica) - Universidade Federal do Paraná, Curitiba, 2004.

3. SILVA, C. D.; FREIRE JUNIOR, R. C. S.; AQUINO, E. M. F. Análise de mecanismo de danos em compósitos híbridos, tipo sanduíche utilizando fibras naturais. Anais: Congresso Brasileiro de Engenharia e Ciências dos Materiais, Porto Alegre, 2004.

4. ALVES, C.; FERRÃO, P.M. C.; SILVA, A. J.; REIS, L. G.; FREITAS, M.; RODRIGUES, L. B.; ALVES, D. E. Ecodesign of automotive components making use of natural jute fiber composites. Journal of Cleaner Production, v.18, p.313-327, 2010.

5. DE ROSA, I. M.; SANTULLI, C.; SARASINI, F. Mechanical and thermal characterization of epoxy composites reinforced with random and quasiunidirectional untreated Phormium tenax leaf fibers. Materials \& Design, v.31, p.2397-2405, 2010.

6. BAKARE, I. O.; OKIEIMEN, F.E.; PAVITHRAN, C.; ABDUL KHALIL, H.P.S.; BRAHMAKUMAR, M. Mechanical and thermal properties of sisal fiber-reinforced rubber seedoilbased polyurethane composites. Materials \& Design, v.31, p.4274-4280, 2010.

7. WAMBUA, P.; IVENS, J.; VERPOEST, I. Natural fibres: Can they replace glass in fibre reinforced plastics? Composites Science and Technology, v. 63, p. 1259-1264, 2003.
8. CARVALHO, K.C.C.; MULINARI, D.R.; VOORWALD, H.J.C.; CIOFFI,

M.O.H. Chemical modification effect on the mechanical properties of HIPS/ coconut fiber composites. BioResources, v.5 (2), p.1143-1155, 2010.

9. NIRMAL, U.; YOUSIF, B. F.; RILLING, D.; BREVERN, P. V. Effect of betelnut fibres treatment and contact conditions on adhesive wear and frictional performance of polyester composites. Wear, v.268, p.1354-1370, 2010.

10. MULINARI, D. R.; VOORWALD, H. J. C.; CIOFFI, M. O. H.; DA SILVA, M. L. C. P.; LUZ, S. M. Preparation and properties of HDPE/sugarcane bagasse cellulose composites obtained for thermokinetic mixer. Carbohydrate Polymers, v.75, p.317-320, 2009.

11. IBRAHIM, M. M.; DUFRESNE, A.; EL-ZAWAWY, W. K.; AGBLEVOR, F. A. Banana fibers and microfibrils as lignocellulosic reinforcements in polymer composites. Carbohydrate Polymers, v.81, p.811-819, 2010.

12. MULINARI, D. R. Comportamento térmico, mecânico e morfológico dos compósitos de polietileno de alta densidade reforçados com fibras de celulose do bagaço de cana-de-açúcar. Tese (Doutorado em Engenharia Mecânica) - Universidade Estadual Paulista, Guaratinguetá, 2009.

13. CHOW, C. P. L.; XING, X. S.; LI, R. K. Y. Effect of water absorption on the mechanical properties of hemp fibre reinforced unsaturated polyester composites. Composites Science and Technology, v.67, p.1674-1683, 2007.

14. MULINARI, D. R.; VOORWALD, H. J. C.; CIOFFI, M. O. H.; DA SILVA, M. L. C. P.; ROCHA, G. J. M. Surface modification of sugarcane bagasse cellulose and its effect on mechanical and absorption properties of sugarcane bagasse cellulose/HDPE composites. Bioresource, v.5, p.661-671, 2010. 
15. STARK, N. Influence of moisture absorption on mechanical properties of wood flour polypropylene composites. Journal Thermoplastics Composites Materials, v.14, p.421-432, 2001.

16. DA SILVA, R. V.; AQUINO, E. M. F.; RODRIGUES, L. P. S.; BARROS, A. R. F. Desenvolvimento de um compósito laminado híbrido com fibras natural e sintética. Revista Matéria, v.13, p. 154 - 161, 2008.

17. RAY, D.; SARKAR, B.K.; BOSE, N.R. Impact fatigue behaviour of vinylester resin matrix composites reinforced with alkali treated jute fibres. Composites Part A v.33; p.233-241, 2002.

18. YUANJIAN, T.; ISAAC, D.H. Impact and fatigue behaviour of hemp fibre composites. Composites Science and Technology v.67, p.3300-3307, 2007.

19. GERRARD, J.; KANDLIKAR, M. Is European end-of-life vehicle legislation living up to expectations? Assessing the impact of the ELV Directive on 'green' innovation and vehicle recovery. Journal of Cleaner Production, v.15, p.17-27, 2007.

20. MOHANTY, A.K.; WIBOWO, A.; MISRA, M.; DRZAL, L.T. Effect of process engineering on the performance of natural fiber reinforced cellulose acetate biocomposites. Composites Part A, v.35, p.363-370, 2004.

21. FERREIRA, A.; LEBRÃO, G. W.; NAGASHI, L.; SU, M. Y.; HANNA, R. K.; LEBRÃO, S. M. G.; VICENTINI, T. Avaliação de compósito de poliéster e fibra de cana de açúcar. Congresso Brasileiro de Engenharia e Ciência dos Materiais, Foz do Iguaçu, 2006.
22. SREEKUMAR, P.A.; ALBERT, P.; UNNIKRISHNAN, G.; JOSEPH, K.; THOMAS, S. Mechanical and water sorption studies of ecofriendly banana fiber-reinforced polyester composites fabricated by RTM. Journal of Applied Polymer Science, v.109, p.1547-1555, 2008.

23. SREEKUMAR, P.A.; JOSEPH, K.; UNNIKRISHNAN, G.; THOMAS, S. A comparative study on mechanical properties of sisal-leaf fibre-reinforced polyester composites prepared by resin transfer and compression moulding techniques. Composites Science and Technology, v.67, p.453-461, 2007.

24. AMICO, S. C.; ANGRIZANI, C. C.; DRUMMOND, M. L. Influence of the Stacking Sequence on the Mechanical Properties of Glass/Sisal Hybrid Composites. Journal of Reinforced Plastics and Composites, v.29, p.179-189, 2010.

25. SCHMIDT, T. M.; GOSS, T. M.; AMICO, S. C. Permeability of Hybrid Reinforcements and Mechanical Properties of their Composites Molded by Resin Transfer Molding. Journal of Reinforced Plastics and Composites, v.28, p.2839-2850, 2009.

26. CICALA, G.; CRISTALDI, G.; RECCA, G.; ZIEGMANN, G.; EL-SABBAGH, A.; DICKERT, M. Properties and performances of various hybrid glass/ natural fibre composites for curved pipes. Materials and Design, v.30, p.2538-2542, 2009.

\section{Endereço para Correspondência:}

Daniella Regina Mulinari daniella.mulinari@foa.org.br Departamento de Engenharia

Centro Universitário de Volta Redonda - UniFOA Campus Olezio Galotti

Av. Paulo Erlei Alves Abrantes, $n^{\circ} 1325$

Três Poços - Volta Redonda - RJ

CEP: 27240-000 